\title{
Germanica
}

\section{Révolutions, tournants de l'histoire et messianisme chez Heinrich Graetz et Moses Hess}

Revolutionen, Geschichtswende und Messianismus bei Heinrich Graetz und Moses Hess

Jean-Marie Delmaire

\section{OpenEdition}

Journals

Édition électronique

URL : http://journals.openedition.org/germanica/2525

DOI : $10.4000 /$ germanica. 2525

ISSN : 2107-0784

Éditeur

Université de Lille

Édition imprimée

Date de publication : 31 décembre 1989

Pagination : 27-41

ISSN : 0984-2632

Référence électronique

Jean-Marie Delmaire, «Révolutions, tournants de l'histoire et messianisme chez Heinrich Graetz et Moses Hess », Germanica [En ligne], 6 | 1989, mis en ligne le 28 novembre 2014, consulté le 06 octobre 2020. URL : http://journals.openedition.org/germanica/2525; DOI : https://doi.org/10.4000/ germanica. 2525

Ce document a été généré automatiquement le 6 octobre 2020.

(c) Tous droits réservés 


\title{
Révolutions, tournants de l'histoire et messianisme chez Heinrich Graetz et Moses Hess
}

\author{
Revolutionen, Geschichtswende und Messianismus bei Heinrich Graetz und
}

Moses Hess

Jean-Marie Delmaire

Parmi les écrivains juifs de langue allemande qui se sont intéressés aux révolutions de 1789 et de 1848, il faut réserver une place à part à l'historien Heinrich Graetz (1817-1891) et à l'inclassable Moses Hess, journaliste, militant socialiste, et précurseur du sionisme (1812-1875) ${ }^{1}$. Graetz, juif pratiquant revenu des tentations de la Réforme qui sévissait alors dans le judaïsme allemand, chercha longtemps sa voie avant d'élaborer une philosophie de l'histoire juive dans sa Konstruktion der jüdischen Geschichte (1846) et de l'illustrer ensuite dans sa monumentale Geschichte der Juden en onze volumes, publiée durant vingt-cinq ans pendant son professorat au séminaire théologique de Breslau. Hess, compagnon de Marx et auteur du célèbre Roter Katechismus für das deutsche Volk, élabora dès ses premiers écrits une conception à la fois messianique et révolutionnaire de l'histoire, condensée dans Die heilige Geschichte der Menschheit (1837). Les deux auteurs, d'opinions politiques différentes, présentent pourtant bien des points communs et se lièrent d'une solide amitié. Ils allaient tous deux à contre courant des conceptions dominantes du judaïsme allemand, réforme ou néo-orthodoxie, science du judaïsme non engagée, intégration bourgeoise et patriotisme : à l'opposé, ils préconisaient une religion de tradition sans trahison mais sans sclérose, une science engagée dans le maintien de la nationalité juive, entre le processus d'assimilation engagé par la Révolution française et le processus d'exclusion engendré par la réaction allemande. Tous deux abhorrent l'Allemagne, ils en présentent une vision critique et lui opposent la France issue des Lumières et de la Révolution, qui ouvre un nouvel âge pour l'humanité entière. Unis par le combat pour la survie du judaïsme, Graetz et Hess choquèrent les juifs allemands par leur nationalisme juif hautement proclamé et par l'aspect polémique de leur œuvre. Rejetés 
par ce public qu'ils méprisaient, ils en trouvèrent tardivement un autre dans la jeunesse juive d'Europe de l'Est. En particulier, les traductions et abrégés de l'Histoire de Graetz nourrirent le nationalisme juif durant plusieurs décennies. Les révolutions de 1789 et de 1848 tiennent une grande place dans leurs écrits et dans les journaux et les lettres, car ils y voient des tournants importants dans l'histoire du monde.

\section{La révolution vécue : 1848}

2 Les années 1840 furent difficiles pour Graetz sur le plan intellectuel et financier. C'est pourtant durant cette période qu'il élabore sa théorie de la structure de l'Histoire juive, qui constitue la première théorisation de cette histoire par les juifs eux-mêmes, et donc la récupération d'une identité dont les autres se sont emparés et qu'ils ont déformé. On en pressent l'élaboration dès 1840 , quand il décrit dans son journal la pétrification des grands courants de pensée antiques à l'époque contemporaine. En terminant sa Konstruktion, il témoigne d'un intérêt soudain envers la politique contemporaine, dont il vient de trouver la clé dans la longue perspective de l'Histoire, passée par trois périodes, «Das politisch-soziale... das religiöse... das theoretischphilosophische» dont les passages sont les deux exils². La troisième période, diasporique, semble aboutir à une réunification des trois caractéristiques, à la suite des Lumières et des soubresauts contemporains. Graetz suivit avec passion les débats sur les juifs à la Diète prussienne en 1847 ; quand la révolution éclata en France puis en Europe, il consacra à ce sujet la majeure partie de son journal intime de 1848: brusquement, il passe de son sort personnel à celui de l'Europe où se déroulait « l'histoire universelle en un bref laps de temps $»^{3}$. Il décrit avec enthousiasme la France gouvernée par un poète, un ouvrier et un juif ${ }^{4}$, la Prusse affolée, la Pologne qui vibre d'espoir, les trônes qui vacillent, puis avec fascination le sursaut de la réaction face aux peuples qui ne vont pas jusqu'au bout de la logique d'une prise du pouvoir. Graetz analyse les trois formes de révolution qui se conjuguent en Europe : chute d'un système paternaliste corrompu, aspirations des nationalités opprimées, lutte contre l'inégalité sociale. Il s'intéresse particulièrement à la chute des États cléricaux et à la fin de la papauté, qu'il juge prochaine (ce sera aussi le point de départ de l'œuvre nationaliste de Hess). L'élection de Louis-Bonaparte marque-t-elle la fin d'une tragi-comédie, « ou bien l'histoire universelle a-t-elle gagné ses quartiers d'hiver? » se demande-t-il, en jugeant sévèrement le renoncement de la France :

En France, la bêtise est au sommet, la combine au ministère et la médiocrité au parlement ${ }^{5}$.

C'est une déception d'amoureux déçu, rare chez lui. En compensation, Graetz cite tout un poème de sa fiancée à la gloire de la révolution et du peuple ${ }^{6}$. La révolution qui voulait l'impossible s'est rendue impossible d'elle-même, mais elle est une semence pour l'avenir. Cependant, Graetz est rempli d'amertume à cause de l'échec conjoint des démocraties et des nationalités où il voit une "époké » au sens grec du terme, un tournant de l'histoire. Il conclut cette année 1848 dans le style qui captivera les lecteurs de son Histoire :

So will ich auch das merkwürdige Jahr zum Abschluss bringen! Wie viel Hoffnungen verschüttet, wie viel Träume vergraben? Auf dem ganzen Erdkreis triumphirt der Hochmuth des Königsthums, die Engherzigkeit des Mammons, der Trotz der Soldateska, die Scheinheiligkeit u. Verschmitztheit der Pfaffen; u. die wahren Freunde der Menschheit überall geächtet, verfolgt u. Eingekerkert oder gar 
ermordet. Die rote Fahne ist geröthet von dem Blut ihrer Träger [...] Bald werde auch ich gezwungen sein, den Wanderstab zu ergreifen ${ }^{7}$.

4 À la suite de 1848 , l'historien retourne vers son propre peuple, comme quelqu'un « qui a cru à tort qu'il avait gagné à la loterie » de l'histoire universelle. C'est dans le déroulement de l'histoire du plus vieux des peuples qu'il cherchera désormais le sens de l'universel.

\section{La révolution théorisée : 1789}

Graetz avait suivi en 1842 à Breslau un enseignement sur la Révolution française, mais il s'y intéressa beaucoup plus tard, en arrivant au onzième volume de son œuvre monumentale. Conformément à sa conception du développement historique, il écrivit sur cet événement final avant de rédiger les deux premiers volumes sur l'historique biblique, puisque c'est par l'étude des origines qu'il trouverait le principe fondamental qui avait permis au judaïsme de traverser les siècles. Longtemps, Graetz n'attribua pas une importance exceptionnelle à la grande Révolution, contrairement à Hess. Lorsqu'il relate dans son Journal sa visite à la place de la Bastille en 1856, il écrit :

Passé à la place de la Bastille, où la libération du genre humain des tyrans médiévaux et barbares eut son beau début. Sur la place se dresse la colonne de juillet, symbole de la falsification de la liberté, de la filouterie diplomatique du constitutionalisme, avec la liberté à la bouche et la servilité au cœur ${ }^{8}$.

6 À cette époque, il se montre plus sensible à la vision d'un cèdre parisien qui évoque pour lui le pays de la Bible. Cependant, l'étude de l'émancipation des juifs le pousse à accorder une plus grande importance à la Révolution de 1789. Le chapitre qui la décrit s'ouvre par une description quasi-messianique :

La Révolution française fut vraiment, selon l'expression du prophète, «le jour du Seigneur où les orgueilleux furent abaissés et les humbles relevés ». Parmi tant d'injustices qu'elle répara, elle mit aussi fin à cette iniquité révoltante qui, depuis tant de siècles, faisait considérer les Juifs comme les parias des nations européennes. Ce que Mendelssohn ne croyait possible que dans un avenir lointain, ce que les défenseurs des Juifs, Dohm et Diez, n'osaient exprimer que sous forme de vœu, la France le réalisa avec une merveilleuse rapidité9.

7 Cependant, après ce brillant préambule, Graetz décrit la Révolution de façon purement factuelle, en insistant sur les personnalités et sur l'aspect législatif et intellectuel : il parle d'un drame et de ses acteurs. L'ancien ennemi des rois reconnaît honnêtement les mérites de Louis XVI dans l'émancipation des juifs, qu'il avait préparée, et déplore les excès de la plèbe :

On sait que la Révolution débuta par la prise de la Bastille. Les excès du peuple de Paris furent imités sur bien des points en France, où des châteaux furent brûlés, des couvents détruits et des nobles maltraités ou tués ${ }^{10}$.

8 Tout en admettant que les juifs ont peu souffert de la Terreur, Graetz accuse la Révolution de deux fautes: les mesures anti-juives de la période du fanatisme et l'exécution du roi. En fait, la Révolution intéresse peu l'historien en tant qu'événement français, mais retient son attention par ses conséquences européennes bénéfiques - là où 1848 échouera : "partout où pénétraient les héroïques soldats français, les Juifs étaient émancipés ${ }^{11}$ (cette phrase est écrite au plus fort de la crise franco-allemande). L'Europe se partage entre une zone éclairée qui accepte l'influence française, et la zone sombre des pays germaniques où subsistent exclusion et préjugés. Pour comprendre 
l'évaluation positive de l'œuvre française, il faut donc examiner les deux accusations de Graetz contre l'Allemagne : ce pays est un reste de Moyen Âge dans l'Europe éclairée, et ses juifs ont trahi Mendelssohn, le père des Lumières.

\section{L'envers de la Révolution : I'Allemagne}

9 Une petite phrase donne la clé du retard de l'Allemagne dans l'émancipation des juifs malgré Lessing et Dohm :

On eût dit que les Allemands cherchaient à se consoler de l'asservissement dans lequel les tenaient le clergé et l'État en humiliant et en maltraitant les Juifs ${ }^{12}$.

10 Le journal de Graetz comme les lettres de Hess sont remplis d'attaques virulentes contre l'Allemagne bouffie de suffisance et bardée de bigoterie qui prétend ressusciter le Saint Empire. L'historien, qui accorde une grande place aux particularités psychologiques de chaque peuple, s'exclame, à propos des Allemands: "Dieu quel peuple lamentable, sans enthousiasme, sans poésie, sans chaleur ». On dresserait sans peine un florilège d'épithètes malsonnantes en de multiples langues contre l'Allemagne et surtout la Prusse : son patriotisme est «ein Eunuchen Kitzel», le peuple est «gens abjecta et vilis» ou en hébreu «'am nevel», et l'ouvrier allemand est encore plus philistin que le bourgeois. Graetz s'en prend surtout aux universitaires, ainsi qu'aux dirigeants socialistes d'origine juive et aujourd'hui apostats ${ }^{13}$. L'Allemagne est l'antithèse de la France des Lumières, parce que le cléricalisme chrétien y pèse de tout son poids, et que l'idéalisme qui marque ce peuple lui ôte tout sens de l'évolution historique.

11 Exilé à Paris, Hess taxe de fourberie le discours humaniste allemand comme le discours socialiste. Ce peuple, défini par sa conscience raciale exclusive, est celui de la dualité tranchée et du manichéisme politique. L'absolutisation du sol et de la race, «l'orgueil sans fondement de la race allemande qui méprise le droit des peuples à disposer d'euxmêmes " constituent l'antithèse du judaïsme "religion de l'Histoire ». Mais elle est aussi l'antithèse de la France émancipatrice et assimilatrice au bon sens du terme. C'est le pays réactionnaire par excellence, qui a pour but véritable la destruction de la Révolution, même lorsqu'il semble évoluer vers la démocratie. La France, elle, garde quelque chose de l'idéal révolutionnaire même dans la dictature ou les orgies de Napoléon III : le peuple ne s'y trompe pas ${ }^{14}$. Hess aussi croit aux caractéristiques innées des peuples, et les métaphorise : la France est le cœur, l'énergie créatrice, le vouloir ; l'Allemagne est le cerveau, elle cherche la vérité idéale et l'absolu religieux. Hess développe cette opposition dans une perspective socialiste dans L'Histoire sacrée de l'humanité et plus encore dans une visée nationaliste dans Rome et Jérusalem: seule la France peut appliquer l'idéal à la réalité :

Mais les Français, soldats de la civilisation moderne, brisent la domination des barbares, roulent de leurs bras d'Hercule les pierres des tombeaux des peuples endormis et ces peuples se réveillent ${ }^{15}$.

12 Tantôt les deux auteurs échangent leurs certitudes antigermaniques, tantôt ils les tempèrent: Hess, sensible à la valeur des personnes, les défend parfois devant Graetz; celui-ci, plus lucide devant l'évolution politique, exprime des réserves sur les régimes français qui permettent une renaissance du cléricalisme et de l'aristocratie ${ }^{16}$. 


\section{La révolution juive trahie : Mendelssohn et ses disciples}

13 Chez Graetz, qui réagit à la déformation hégélienne d'un judaïsme confiné dans le passé oriental, la division des chapitres de l'histoire est aussi importante que leur contenu, sinon plus. La dernière période, intitulée "Le relèvement ", ne commence pas avec la Révolution française, mais avec Mendelssohn: celui-ci accomplit dans l'âge «spéculatif » ce que Socrate avait commencé dans l'âge politico-religieux. La gravure de Laurens représentant les deux philosophes face à face illustre le surnom souvent appliqué au philosophe de Dessau, mais la conception graetzienne va plus loin qu'un simple rapprochement de circonstance : l'homme qui inaugure une époque (David, Esdras, les Maccabées, Saadia, Maïmonide, Mendelssohn) exerce une fonction particulière d'initiateur et de révélateur :

Ein Gedanksystem gehört aber nicht ganz oder teilweise dem Schöpfer desselben an, es ist ein Produkt der Zeit, und der Denker hat dabei oft nur die Funktion, dass er die zerstreuten Strahlen der Idee in einen Brennpunkt sammelt ${ }^{17}$.

14 Situé au terme de la diaspora, avant le rétablissement espéré de la nationalité sur sa Terre, Mendelssohn est pour Graetz l'émanation de l'esprit de la diaspora, le " contrepoint de l'histoire juive » :

Moïse Mendelssohn, qui contribua pour une si large part au relèvement du judaïsme, présentait en quelque sorte, dans sa personne, l'image même de son peuple. Petit, contrefait, un peu gauche, il était d'un extérieur assez déplaisant ; mais ce corps, d'apparence frêle et débile, était animé d'une intelligence vigoureuse à laquelle nul effort ne coûtait pour arriver à la vérité ${ }^{18}$.

De même le peuple juif, déformé par les persécutions, se redresse dès qu'on lui montre la Lumière. Cette louange sans réserves fut critiquée, mais Graetz en tirait un double confirmatur: le premier, assez inattendu, est la justification de son nationalisme à travers la conception du judaïsme comme «législation révélée » et non "religion révélée ». Pour Graetz, Mendelssohn formule cette distinction non pas pour «épurer » le judaïsme, mais parce qu'il a instinctivement saisi que le juif est juif quand il est fidèle aux deux temps de son histoire, le politique et le religieux: «der Bürgerdienst ist Gottesdienst ${ }^{19}$. La seconde confirmation est celle de sa juste colère contre les juifs allemands qui auraient trahi Mendelssohn en le germanisant et en le christianisant : ils se sont contentés d'une conception allemande de la philosophie spéculative et de la science pure, alors que la mission et le génie du judaïsme sont de faire passer les vérités éternelles dans le domaine de l'histoire en évolution; ils ont reflété l'égoïsme allemand en réglant leur sort individuel par la conversion pour une promotion sociale, au lieu de rester fidèles au peuple et à la nation.

Ce n'est donc pas l'Allemagne qui porte à son point culminant l'œuvre de Mendelssohn. L'application juive des Lumières se fait en France, et l'on comprend le grand intérêt porté par Graetz à la société juive qui incarna le mieux les idéaux de 1789, comme l'a bien analysé G. Weill: l'Alliance Israélite Universelle, créé à Paris en 1860 pour rayonner à travers le monde ${ }^{20}$. Graetz échangea avec elle une correspondance suivie, l'Alliance finança la traduction hébraïque de son Histoire. Leur accord ne se limita pas aux principes républicains, mais engloba aussi une certaine conception de la nationalité, car l'Alliance observait alors avec intérêt certains projets " palestinophiles ». Graetz lui fournit des renseignements après son voyage en Terre 
Sainte, il collabora aussi à son action pour les victimes des pogromes à partir de 1881, en remarquant au passage que les juifs allemands, timorés et égoïstes, étaient incapables de comprendre l'idéal humanitaire de l'Alliance ${ }^{21}$.

\section{L'accomplissement de l'évolution et de la Révolution : la renaissance d'Israël}

17 Quand Hess présenta en Allemagne le manuscrit de son Wiedergeburt Israels, rédigé fiévreusement en 1860-1861, il ne rencontra que scepticisme et raillerie, sauf chez Graetz qui l'invita à Breslau et fit éditer son livre sous le titre qu'il lui suggéra Rom und Jerusalem. Ainsi le messianisme particulier d'Israël entrait-il en conjonction avec le messianisme historique universel inauguré par la Révolution et qui semblait trouver alors son achèvement par l'écroulement attendu de la papauté et la formation de l'Italie.

L'ère messianique, c'est notre époque ; elle a commencé à poindre avec Spinoza et elle est apparue dans l'histoire avec la Révolution française. La Révolution française amena la renaissance des peuples qui doivent leurs sentiments aux judaïsme ${ }^{22}$.

L'historien israélien Michael Graetz a bien montré dans son article «Anfänge der modernen jüdischen Geschichtschreibung» que la «construction » de l'histoire juive par Graetz était destinée à la faire échapper à la théologie chrétienne comme à la philosophie hégélienne. L'historien Graetz fait sortir son peuple diasporique de la tombe où l'enfermait le christianisme avant que l'Histoire ne le réintègre au concert des nations. Son rôle est d'expliquer le début de cette négation et d'annoncer sa fin: c'est bien ce début, la naissance et la séparation du christianisme, que Hess choisit de traduire en français sous le titre Sinaï et Golgotha en 1867, de même qu'il cita très longuement dans son Rome et Jérusalem l'analyse de Jésus par Graetz ${ }^{23}$. Tous deux cherchent à prouver l'unité organique de l'histoire juive (contre la conception chrétienne du Nouvel Israël qui fait du judaïsme postbiblique un résidu) et par corollaire la spécificité radicale du judaïsme («Die Hebräer, die Nachkommen Ewers, sind nicht bloss gradativ, sondern generisch von andern Völkern verschieden $\left.{ }^{24}\right)$. Dès l'origine, la mission propre du judaïsme est l'élévation spirituelle et matérielle de l'humanité, qui aboutit, au terme de la période philosophique, en «eine religiöse Staatsverfassung». La restauration de la nationalité juive est donc beaucoup plus que la simple réparation d'une injustice : c'est l'aboutissement d'une période courte, celle de la Révolution, puisque chaque âge a sa tendance propre, mais surtout l'aboutissement de toute l'histoire, de l'évolution depuis les origines, ce que Moses Hess appelle «le sabbat de l'histoire». Hess le décrit en termes quasi biologiques, en une espèce d'ontogenèse du corps national, et Graetz en termes de philosophie de l'histoire ${ }^{25}$.

En quoi la Révolution entraîne-t-elle cette restauration messianique ? Jusque 1789, la religion juive était la seule à être une religion de l'histoire, nationale et universelle à la fois. Depuis la Révolution, selon Hess, cette double essence nationale et universelle « est le germe de l'organisation future de la société ». La France et les peuples qui l'ont suivie sont donc devenus "émules et fidèles alliés des Juifs ", c'est donc la marche finale vers l'unité organique de l'humanité dans la diversité des nations libérées ${ }^{26}$. Hess ne craint pas de développer d'autres aspects paradoxaux ou différents, sociaux et politiques : la Révolution a détruit le vieux monde féodal en mettant face à face travail et spéculation 
(ou même «le matérialisme et l'idéalisme »), ou encore : la Révolution a décapité la dernière race dominante en décapitant son roi !

L'Allemagne, quant à elle, n'a jamais connu de révolution, ni même de Réforme véritable, selon Graetz; elle reste le témoin figé de l'État féodal, elle ne peut comprendre la France révolutionnaire qu'à travers ses conceptions déformées :

Ils oublient que ce n'est pas l'armée qui a créé la grande Nation, mais au contraire que c'est la grandeur et la force morale de la Nation révolutionnaire qui a créé l'armée française et ses héros ${ }^{27}$.

21 Comme l'accomplissement est encore à venir, il est enveloppé d'incertitude en ce qui concerne ses modalités : victoire souhaitée de la France sur l'Allemagne qui sera alors livrée à la révolution sociale ; réitération accélérée de l'histoire avec le nouveau Déluge qu'est 1789, le nouvel Attila qu'est Napoléon, la nouvelle Jérusalem que sera la Révolution instaurée ; ou encore vision d'une complémentarité pacifique retrouvée des contraires. Hess, qui multiplie ces chimères, pense que la France lèvera bientôt les deux derniers obstacles, la Prusse et la papauté. Graetz ne partageait pas cette fougue : ce n'est pas la réconciliation de la volonté française et de la contemplation allemande qui importaient, mais l'aboutissement d'une logique interne à l'histoire juive.

On comprend aisément que de telles conceptions furent violemment combattues, au nom de l'internationalisme et de la lutte antireligieuse par les anciens compagnons de Hess, au nom d'une fonction sociale purement morale et d'une pratique cultuelle purement individuelle par les assimilés, au nom d'une vision scientifique de l'histoire, chez les tenants de la science du judaïsme. Ces derniers réservèrent à Graetz sarcasmes et humiliations, dans leurs recensions ou par leur refus de l'accepter dans la commission pour l'histoire du judaïsme en Allemagne, en 1885 :

Es handelt sich unserem Unternehmen nicht um Geschichtschreibung sondern um kritische Erforschung der Materialen. Nicht um Conjonctionen um Polemik und Apologetik [...]. Was dann der eizelne Geschichtschreiber nach seinem individuellen Judicium, pectus und Pathos daraus machen will, das bleibt ihm überlassen ${ }^{28}$.

\section{L'influence de Graetz}

23 L'influence immédiate de Hess fut presque nulle : ses anciens compagnons socialistes ne se précipitèrent pas pour recueillir ses nombreux manuscrits, Graetz brûla ses lettres, la presse ne le cita guère, et l'écrivain Lilienblum déclare inutile un projet de traduction en brochure populaire hébraïque de cet auteur dont le nom ne dit rien aux juifs de Russie ${ }^{29}$. Il faudra attendre au début du vingtième siècle sa récupération par le sionisme désireux de s'enraciner dans le passé juif proche ou lointain. Par contre Graetz, quoique boudé par les savants, obtint un succès populaire immédiat et fut un des auteurs juifs les plus lus avant 1914: outre la traduction française par Hess, Die Geschichte der Juden bénéficia de nombreuses rééditions, et de traductions en anglais (1891-1892), en français (1882-1897), en yiddish (1897-1898), en hébreu (1890-1899) et en plusieurs autres langues, ainsi que d'éditions populaires abrégées en allemand, anglais et yiddish. La traduction hébraïque de S.P. Rabbinowitz (Shefer), écrivain et militant nationaliste, présente une importance particulière pour le volume sur l'histoire récente. Elle parut avec un support de groupes russes des Amants de Sion, à la suite de tentatives de récupération de Graetz par les nationalistes de Russie. Au lendemain de la première assemblée palestinophile de Kattowitz (fin 1884), Graetz fut pressenti pour prendre la tête du mouvement, mais il refusa par fatigue, scepticisme, et 
surtout crainte des juifs allemands et des antisémites ${ }^{30}$. Néanmoins, son soixantedixième anniversaire fut célébré avec éclat dans les milieux sionistes et plus tard les notices nécrologiques de 1891 mirent l'accent sur son nationalisme. Quand Rabbinowitz entreprit sa traduction hébraïque, il voulut corriger Graetz sur trois points : compléter ses chapitres sur les juifs russes, diminuer l'importance de Mendelssohn et accentuer l'aspect nationaliste. L'auteur le lui interdit expressément, mais le traducteur passa outre à ces consignes après la mort de Graetz. L'Alliance israélite finança cette œuvre, dont on peut suivre la progression par la correspondance conservée dans ses archives. Shefer fait d'une histoire de portée universelle une histoire nationale, où l'hébreu devient un but et non un outil, et où la structure de l'histoire cède le premier rang à «l'action des grands hommes qui font l'histoire " $^{31}$. Sous cette forme, Graetz fournit des armes au sionisme qui adopta un schéma plus simple de l'histoire en opposant la gloire passée, la décadence de l'exil, et la restauration de l'État juif. Motzkin, alors dirigeant des étudiants sionistes berlinois, assigne comme tâche essentielle au nationalisme «die Anerkennung der Vergangenheit, die Hoffnung auf die Zukunft», et affirme ailleurs que l'histoire était le trésor de la jeunesse qui lisait Graetz comme un roman ${ }^{32}$. Leur source de compréhension du présent n'était pas son analyse de l'émancipation mais sa peinture fascinante de l'époque des rois Macchabées vainqueurs de l'héllénisme: Graetz fut utilisé dans les "fêtes des Macchabées ", nouvelle forme donnée dans les cercles nationalistes à la fête de Hannouka et véritable fête nationale de la liberté. D'autres reconstructions de l'histoire en hébreu, yiddish, et allemand ont été étudiées par moi-même et, pour la période suivante par S. Almog dans Zionism and history ${ }^{33}$. Les principales conceptions qui s'affrontent sont celles du volontarisme d'une création de l'histoire, d'une nécessité historique externe (suite de l'émancipation), ou interne (l'esprit du peuple). L'article qui illustre le mieux le glissement des idées de Graetz est peut-être celui de l'écrivain hébraïque Lilienblum, «Le temps et son rapport au peuple ", où il utilise la réhabilitation de l'histoire du peuple comme unité organique pour y ajouter un second volet : si le peuple est le produit de l'histoire, l'homme est le produit de la nature. Le peuple d'Israël n'est pas un anachronisme dans le temps, comme le croient les chrétiens ; les historiens ont prouvé le contraire. Il est par contre un être anormal dans l'espace, parce que l'homme juif est hors de son lieu naturel. Reconnu comme "doté d'histoire ", le peuple juif doit donc être aussi « doué d'État " pour que son émancipation ne soit pas une assimilation ${ }^{34}$.

Ainsi, l'effort de Graetz pour faire rentrer le judaïsme au premier rang des «peuples historiques » combiné avec sa certitude qu'il était « non une religion du présent, mais de l'avenir" servit-il un sionisme dont il n'apprécia pas tous les signes précurseurs. Bientôt dépassé par Dubnow et d'autres historiens, il demeura cher aux lecteurs d'Europe de l'Est comme l'était Mapou, le créateur du roman historique hébraïque, par l'amour du peuple opprimé qu'il chérissait et auquel il rendait sa dignité. 


\section{NOTES}

1. - Sur Graetz, nous disposons d'une biographie ancienne par Josef Meisl, Heinrich Graetz, parue en 1917 à Berlin pour le centième anniversaire de sa naissance, mais il est mieux connu depuis l'édition de ses Tagebuch und Briefe par Reuven Michael, Tübingen, 1977. Sur Hess, deux œuvres d'E. Silverner surpassent les autres: Moses Hess, Geschichte seines Lebens, Leiden, 1966, et son édition de Moses Hess Briefwechsel, Amsterdam, 1959.

2. - H. Graetz, Die Konstruktion der jüdischen Geschichte, eine Skizze (édition Schocken, Berlin, 1936), p. 96 : Tagebuch, p. 112.

3. - Tagebuch., p. 175.

4. - Ibid., p. 176 (Lamartine, l'ouvrier Albert, Crémieux) ; «Und welch grosse, weltgeschichtliche, zukunftsreiche Begebenheiten, die Throne wankend machen, die ganze Stellung eine Lösung oder Auflösung entgegenführen [...]. Neue Hoffnung wurde erweckt, längst gestorben Wünsche halten Auferstehung, unbekannte Ahnungen durchzucken und durchschauern die Menschheit, jeder fühlt sich grösser, gehobener, berechtiger! Und nun diesen Riesengedanken gegenüber die verzerrte Zwerggestalt der deutschen Regierungen! Die Zensur, dit Polizei, die Militärwirtschaft, die ganze Misere einer gekünstelten Regierung, welche ihre Macht auf die verschrobenen Ideen des christlichen Staates stützen muss!».

5. - Ibid., p. 179 et 183.

6. - Ibid., p. 184-186.

7. - Ibid., p. 193.

8. - Ibid., p. 216.

9. - Histoire des Juifs, éd. française de 1897, t. V, p. 306.

10. - Ibid., p. 309.

11. - Ibid., p. 316-318 (citation, p. 318).

12. - Ibid., 319. Graetz n'hésite pas à dénoncer Goethe et Fichte.

13. - Tagebuch, p. 133, p. 242-243, p. 250 (à Hess), 270 (id.) ; p. 240 (id.).

14. - Silerner, Moses Hess, p. 283 sq. et 358 sq. ; Rome et Jérusalem, p. 63 (trad. française de 1981, éd. Albin Michel) ; ibid., p. 92 ; cf. Graetz Tagebuch und Briefe, p. 267 : «Die Franzosen tanzen doch wenigstens inzwischen den Cancan und aus den Orgienkann eher eine Revolution hervorgehen als aus der sittlicher Entrüstung».

15. - Rome et Jérusalem, p. 99 : sur les développements antérieurs chez Hess, voir J. Fraenkel, Prophecy and politics, Cambridge, 1981, p. 7 sq.

16. - Cf. Graetz, op. cit., p. 250 , p. 322, après la guerre de 1870 : «Sie haben recht, dass die Deutschen nach solchen Katastrophen caput gewesen wären, und man muss die Zähigkeit der Franzosen bewundern. Aber der Hass der Parteien miteinander, die kleiniche Schadenfreude, die jeder empfindet, wenn ihrer Gegnerin eine Malice an denKopf geworfen wird, und namentlich die Buhlerei mit dem Clericalismus, das sieht nach Verjüngung aus?».

17. - Graetz, Konstruktion, p. 72 (citation), ibid., p.90-91, Mendelssohn et Socrate; Moses Mendelssohnn and his Time, exposition à Tel-Aviv, 1979, reproduction de la gravure de Laurens (1819) et buste du philosophe par Tassaert avec l'inscription «Ein weiser wie Sokrates».

18. - Histoire des juifs, tome V, p. 265.

19. - Konstruktion, p. 92.

20. - Georges Weill, «Émancipation et humanisme; le discours idéologique de l'Alliance Israélite Universelle au XIX ${ }^{\mathrm{e}}$ siècle », Les Nouveaux Cahiers, 52, 1978, p. 1-20.

21. - Archives de l'AIU, Paris, Pologne E-L-M et XI b; Meisl, op. cit., Beilage II, III, V; Tabebuch und Briefe, passim, spéc. p. 370 . 
22. - Silberner, Moses Hess, ch. XIV et XVI, 3, et la correspondance publiée dans les ouvrages cités à la note 1. Rome et Jérusalem, p. 107 et p. 158-159 (citation).

23. - M. Graetz, «Anfänge der modernenjüdischen Geschichtsschreibung», LessingHeft, 4, 1985 ; Silberner, op. cit., p. 485 sq. ; Rome et Jérusalem, p. 203-218.

24. - Graetz, Konstruktion, p. 79.

25. - Hess, op. cit., p. 155, aussi p. 71 et p. 255 sq., la longue note VII de Hess. Comparer à Graetz, Konstruktion, p. 8-9 et 92-93.

26. - Hess, op.cit., p. 126 et 144 ; autres conceptions, p. 226-228, 235. Voir aussi l'idée du rapprochement positif des classes bourgeoises et ouvrière en France après la Révolution, à l'opposé de l'Allemagne, dans sa lettre à L'Internationale en 1871, in Silberner, Moses Hess Briefwechsel, p. 612.

27. - D’un article de Hess dans L'espérance, 18/12/1859, cité par Silberner, Moses Hess, p. 380 ; Graetz, lettre à Hess du 15/10/1862 : «Die deutsche oder richtiger germanische Race, welche so recht eigentlich das Mittelalter geschaffen hat, hat diesen Bau so cyclopisch fest gefügt, dass alle Ströme daran unmächtig abprallen», Tagebuch und Briefe, p. 237.

28. - Meisl, op. cit., Beilage VIII, p. 182 ; Graetz, op. cit., p. 310 ; c'est donc logiquement que Hess qualifie Rom und Jerusalem de "germanenfeindliche Schrift» et de "révolutionnaire ", Briefe, p. 418 et 386 .

29. - Archives Centrales Sionistes, Jérusalem, ACS A $24 / 1 \mathrm{~V} / 1, \mathrm{n}^{\circ} 54$ et 58 . Une brochure de 24 pages parut en 1894 et se diffusa mal. Voir aussi l'introduction de Silberner, Briefwechsel.

30. - Israël Klausner, MiKattowitz 'adBazel, vol. I, Jérusalem, 1965, p. 30 sq. Graetz, Tagebuch und Briefe, p. 402 sq (lettres à Pins ker) «weil mein Name, anstatt zu ziehen, Angriffspunkte für rabiate Antisemiten und entjüdischte Juden werden könnte».

31. - Archives de l'Alliance Israélite Universelle, Paris, Pologne E-L-M, dossier S.P. Rabbinowitz : le traducteur y travailla quatorze ans ; citation du 21/11/1894 (de l'hébreu) ; les lettres de Graetz à Shefer ont été publiées par Meisl, annexe VII, refus des changements dans la lettre du 3/1/1888. 32. - Motzkin, in Selbstemanzipation, V, 3 (1/2/1892) et in V. Pilovsky, Nahman Syrkin, Dissertation, Jérusalem, 1974, p. 27.

33. - J.-M. Delmaire, De Hibbat-Zion au sionisme, doctorat, Strasbourg, 1986, p. 808-815 et p. 823-826; Almog, 1987, p. 23 sq.

34. - Lilienblum, in Luah Ahiasaf, II (1894), p. 121 sq. ; III (1895), p. 103 sq. ; en allemand, voir O. Thon, Zur Geschichtphilosophische Begründung des Zionismus, Berlin, 1896 (articles parus dans Ziori).

\section{RÉSUMÉS}

À l'époque de la Révolution de 1848, l'historien Graetz et le militant socialiste Hess ont élaboré une réflexion sur l'histoire universelle qui aboutit à une conception nationale du judaïsme. Ils comprennent les événements de 1789 et le développement des Lumières en milieu juif à la suite de Mendelssohn comme le début d'une nouvelle période de l'histoire, mais aussi comme l'aboutissement de l'histoire universelle, où ils lient la restauration de la nationalité juive et les soubresauts de l'Europe, en opposant fortement l'Allemagne et la France, symboles de deux états successifs de l'humanité, réconciliables seulement dans une perspective messianique. Graetz inspira le nationalisme juif russe et lui fournit des arguments, alors qu'il ne fut guère apprécié en Allemagne. 
Die Reflexion des Historikers Graetz und des Vorkämpfers des Sozialismus Moses Hess zur Zeit der 48er Revolution über die Weltgeschichte mündet in eine nationale Auffassung des Judentums. Sie verstehen die Ereignisse der Französischen Revolution und die bei den Juden verbreiteten Ideale der Aufklärung nicht nur als den Anfang einer neuen geschichtlichen Epoche, sondern auch als das Ergebnis der Weltgeschichte. Sie bringen die Wiederherstellung des jüdischen Nationalgefühls und die Erhebungen in Europa miteinander in Zusammenhang, indem sie Deutschland und Frankreich als Symbole für zwei aufeinanderfolgende, nur in messianischer Perspektive zu versöhnende Stufen der Menschheitsgeschichte entgegensetzen. Graetz beinflußte den jüdischen Nationalismus in Rußland und gab ihm Argumente, während er in Deuschland nicht sehr geschätzt wurde.

\section{AUTEUR}

JEAN-MARIE DELMAIRE

Université de Lille III 